\title{
Gaussian RBF-FD weights and its corresponding local truncation errors
}

\author{
V. Bayona, M. Moscoso \& M. Kindelan \\ Universidad Carlos III de Madrid, Spain
}

\begin{abstract}
In this work we derive analytical expressions for the weights of Gaussian RBFFD formulas for some differential operators. These weights are used to derive analytical expressions for the leading order approximations to the local truncation error in powers of the internode distance $h$ and the shape parameter $\epsilon$.

We show that for each differential operator, there is a range of values of the shape parameter for which RBF-FD formulas are significantly more accurate than the corresponding standard FD formulas. In fact, very often there is an optimal value of the shape parameter $\epsilon^{+}$for which the local error is zero to leading order. This value can be easily computed from the analytical expressions for the leading order approximations to the local error. Contrary to what is generally believed, this value is, to leading order, independent of the internodal distance and only dependent on the value of the function and its derivatives at the node.

Keywords: Gaussian RBF, RBF-FD formulas, optimal shape parameter.
\end{abstract}

\section{Introduction}

Radial basis functions (RBFs) were first used as an efficient technique for interpolation of multidimensional scattered data [7]. Later, it became popular as a truly mesh-free method for the solution of partial differential equations (PDEs) on irregular domains. This application of RBFs was first proposed by Edward Kansa $[12,13]$ and it is based on collocation in a set of scattered nodes. The main advantages of the method are ease of programming and potential spectral accuracy, but its main drawback is ill-conditioning of the resulting linear system.

To overcome this drawback a local RBF method was independently proposed by several authors [15-17]. The method is based on approximating the solution as a linear combination of a set of identical RBFs translated to a set of RBF centers. 
The approximation is local, so it is carried out within a small influence domain instead of a global one. Thus, the resulting linear system is sparse, overcoming the ill-conditioning of the global method, at the cost of losing its spectral accuracy.

The local RBF method can also be considered as a generalization of the classical Finite Difference (FD) method. In the FD method the weights are computed using polynomial interpolation, while in the local RBF method they are computed by fitting an RBF interpolant through a grid point and a small number of its nearest neighbors. Since both, FD and local RBF formulas are identical in form, we will refer to the local RBF method as the RBF finite difference (RBF-FD) method, as in [17].

Many of the RBFs used in practical applications contain a shape parameter that has to be chosen a priori. The accuracy of the approximated solution strongly depends on its value. Thus, the problem of how to select appropriate values for the shape parameter has been of primary concern. In a recent paper [1] we derived analytical approximations to the local approximation error for 1D and 2D differential operators using multiquadrics as RBFs. These formulas were then used to propose efficient algorithms for the selection of either an optimal (constant) value of the shape parameter that minimizes the approximation error [2], or optimal (node dependent) values that minimize the local residual error [3]. In this paper, we carry out a similar analysis to the one performed in [1], but using Gaussians instead of multiquadrics as RBFs. The formulas for the local approximation error that we derive below can then be used to compute the optimal value of the shape parameter (both constant and variable) in a way similar to that used in [2,3].

There are not too much work relating to the RBF-FD method using Gaussians as RBFs. One should mention the work of Flyer and Wright [10] and Davydov and Oanh [8,9] from the application point of view, and the work of Wright [17] and Fornberg and Letho [11] and Boyd and Wang [4] from the analytical point of view.

\section{RBF-FD formulation}

Consider a differential operator $\mathcal{L}[\cdot]$ and a stencil consisting of $n$ scattered nodes $\left\{\mathbf{x}_{1}, \mathbf{x}_{2}, \ldots, \mathbf{x}_{n}\right\}$. For a given node $\mathbf{x}=\mathbf{x}_{j}(1 \leq j \leq n)$, the differential operator can be approximated by the formula

$$
\mathcal{L}\left[u\left(\mathbf{x}_{j}\right)\right] \approx \sum_{i=1}^{n} \alpha_{i} u\left(\mathbf{x}_{i}\right),
$$

where $\alpha_{i}$ are the weighting coefficients. In the standard FD formulation, these weights are computed using polynomial interpolation. In the RBF-FD formulation, RBF interpolants are used instead. Thus,

$$
u(\mathbf{x})=\sum_{i=1}^{n} \lambda_{i} \phi\left(\left\|\mathbf{x}-\mathbf{x}_{i}\right\|\right),
$$


where $\|$.$\| is the euclidean norm and \phi(r)$ is some radial function. The unknown weights $\alpha_{i}$ can be determined by solving the system of linear equations,

$$
\mathcal{L}\left[\phi\left(\left\|\mathbf{x}_{k}-\mathbf{x}_{j}\right\|\right)\right]=\sum_{i=1}^{n} \alpha_{i} \phi\left(\left\|\mathbf{x}_{k}-\mathbf{x}_{i}\right\|\right), \quad k=1, \ldots, n,
$$

which is obtained after substituting (2) in (1) and doing some algebra . Notice that these RBF-FD interpolation formulas are not exact for constants. To guarantee this condition, a constant term ( $\beta$ ) should be added in the RBF interpolant (2). The unknown weights $\alpha_{i}$ are computed by solving the system of linear equations

$$
\left\{\begin{array}{l}
\mathcal{L}\left[\phi\left(\left\|\mathbf{x}_{k}-\mathbf{x}_{j}\right\|\right)\right]=\sum_{i=1}^{n} \alpha_{i} \phi\left(\left\|\mathbf{x}_{k}-\mathbf{x}_{i}\right\|\right)+\mu, \quad k=1, \ldots, n . \\
\sum_{i=1}^{n} \alpha_{i}=0
\end{array}\right.
$$

where $\mu$ is a constant related to $\beta$.

\section{Weights and truncation error}

In this section, we derive analytical expressions for the weights of RBF-FD formulas using Gaussians as RBFs,

$$
\phi\left(\left\|\mathbf{x}-\mathbf{x}_{j}\right\|\right)=\exp \left(-\epsilon^{2}\left\|\mathbf{x}-\mathbf{x}_{j}\right\|^{2}\right),
$$

where $\epsilon$ is the shape parameter. We consider RBF-FD formulas for first and second order derivatives in $1 \mathrm{D}$, and for the Laplacian in $2 \mathrm{D}$, using equispaced nodes. The weights are functions of the internode distance $h$ and the shape parameter $\epsilon$. Contrary to what happened with multiquadrics [1], in which case the weights were written as Taylor series expansions in powers of $h$, for Gaussians it is often possible to write them as short analytical formulas. Using these coefficients, we also derive analytical expressions for the leading term of the local truncation error in the limit $\epsilon h \ll 1$. In the tables we use the notation $O\left(h^{m} P_{n}\left(\epsilon^{2}\right)\right)$ to indicate that the terms that have been neglected are of order $h^{m} \sum_{i=0}^{n} a_{i} \epsilon^{2 i}$, where $a_{i}$ are constants.

To check the validity of the formulas given in this subsection, we use

$$
f(\mathbf{x})=\sin \left(\|\mathbf{x}\|^{2}\right),
$$

as test function. Equations (3) and (4) are used to compute the coefficients needed to approximate the corresponding operator $\mathcal{L}[\cdot]$ at $x_{0}=0.4$ and $\mathbf{x}_{0}=(0.4,0.4)$ in $1 \mathrm{D}$ and 2D, respectively. For each formula, we compute the absolute value of the error as a function of the shape parameter $\epsilon$ and the node distance $h$, and compare it with the leading term of the local truncation error in the limit $\epsilon h \ll 1$. 
Table 1: RBF-FD first derivative.

\begin{tabular}{|c|c|}
\hline \multicolumn{2}{|r|}{ Three nodes } \\
\hline$\alpha_{0}$ & 0 \\
\hline$\alpha_{ \pm 1}$ & $\pm \frac{1}{2} \epsilon^{2} h\left(\operatorname{csch}\left(\epsilon^{2} h^{2}\right)+\operatorname{sech}\left(\epsilon^{2} h^{2}\right)\right)$ \\
\hline$\tau_{3}$ & $\frac{h^{2}}{6}\left(u^{\prime \prime \prime}\left(x_{0}\right)+6 \epsilon^{2} u^{\prime}\left(x_{0}\right)\right)+O\left(h^{4} P_{2}\left(\epsilon^{2}\right)\right)$ \\
\hline \multicolumn{2}{|r|}{ Five nodes } \\
\hline$\alpha_{0}$ & 0 \\
\hline$\alpha_{ \pm 1}$ & $\pm \epsilon^{2} h\left(1+e^{2 \epsilon^{2} h^{2}}\right) \operatorname{csch}\left(3 \epsilon^{2} h^{2}\right)$ \\
\hline$\alpha_{ \pm 2}$ & $\mp \frac{\epsilon^{2} h e^{4 \epsilon^{2} h^{2}}}{\sinh \left(2 \epsilon^{2} h^{2}\right)+\sinh \left(4 \epsilon^{2} h^{2}\right)+\sinh \left(6 \epsilon^{2} h^{2}\right)}$ \\
\hline$\tau_{5}$ & $-\frac{h^{4}}{30}\left(u^{(V)}\left(x_{0}\right)+20 \epsilon^{2} u^{\prime \prime \prime}\left(x_{0}\right)+60 \epsilon^{4} u^{\prime}\left(x_{0}\right)\right)+O\left(h^{6} P_{3}\left(\epsilon^{2}\right)\right)$ \\
\hline \multicolumn{2}{|r|}{ Seven nodes } \\
\hline$\alpha_{0}$ & 0 \\
\hline$\alpha_{ \pm 1}$ & $\pm \epsilon^{2} h\left(e^{3 \epsilon^{2} h^{2}}+2 \cosh \left(\epsilon^{2} h^{2}\right)\right) \operatorname{csch}\left(4 \epsilon^{2} h^{2}\right)$ \\
\hline$\alpha_{ \pm 2}$ & $\epsilon^{2} h e^{2 \epsilon^{2} h^{2}}\left(1+e^{2 \epsilon^{2} h^{2}}+e^{4 \epsilon^{2} h^{2}}\right)$ \\
\hline$\alpha_{+3}$ & $\frac{\epsilon^{2} h e^{9 \epsilon^{2} h^{2}}}{\sinh \left(2 \epsilon^{2} h^{2}\right)+2 \sinh \left(4 \epsilon^{2} h^{2}\right)+2 \sinh \left(6 \epsilon^{2} h^{2}\right)+2 \sinh \left(8 \epsilon^{2} h^{2}\right)+\sinh \left(10 \epsilon^{2} h^{2}\right)+\sinh \left(12 \epsilon^{2} h^{2}\right)}$ \\
\hline$\alpha_{-3}$ & $-\frac{\epsilon^{2} h e^{9 \epsilon^{2} h^{2}} \operatorname{csch}\left(6 \epsilon^{2} h^{2}\right)}{2\left(1+2 \cosh \left(2 \epsilon^{2} h^{2}\right)+\cosh \left(4 \epsilon^{2} h^{2}\right)+\cosh \left(6 \epsilon^{2} h^{2}\right)\right)}$ \\
\hline$\tau_{7}$ & $\frac{h^{6}}{140}\left(u^{(V I I)}\left(x_{0}\right)+42 \epsilon^{2} u^{(V)}\left(x_{0}\right)+420 \epsilon^{4} u^{\prime \prime \prime}\left(x_{0}\right)+840 \epsilon^{6} u^{\prime}\left(x_{0}\right)\right)+O\left(h^{8} P_{4}\left(\epsilon^{2}\right)\right)$ \\
\hline
\end{tabular}

\subsection{First derivative}

Table 1 shows the weights and the corresponding local truncation errors for RBFFD formulas to approximate the first derivative in 1D. Exact expressions are given for 3, 5 and 7 equispaced nodes. The results for 3 and 5 nodes are in agreement with those previously derived in Appendix A of reference [4].

Figure 1 shows the corresponding error (solid line) for $n=3,5,7,9$, and compares it with the approximate error given by the formulas in Table 1 (dashed line). Notice that the agreement is excellent up to the point where the linear system to numerically compute the weights (3) becomes ill-conditioned and round-off errors deteriorate the accuracy of the numerical solution. The left part of Figure 1 shows the absolute value of the error as a function of the shape parameter for $h=0.05$. The accuracy increases with decreasing $\epsilon$. For small $\epsilon$ (flat RBFs) it is 

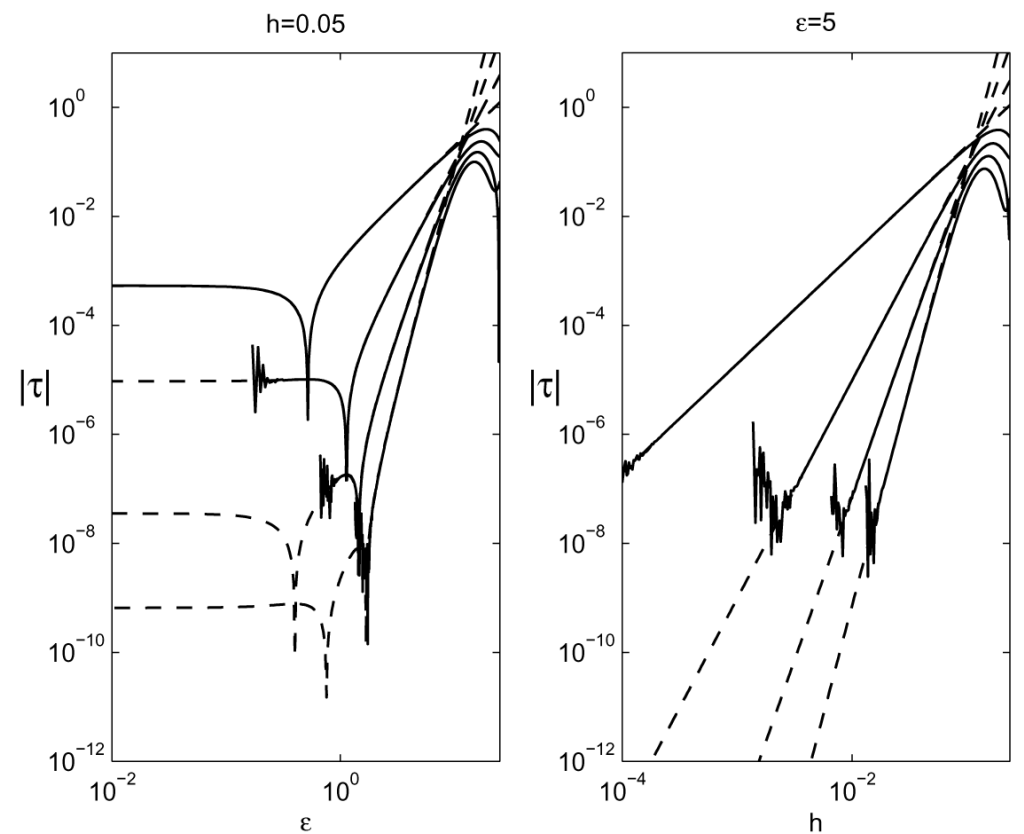

Figure 1: Local truncation error $\tau$ for the RBF-FD first derivative as function of $\epsilon$ (left side) and $h$ (right side) using structured stencils with $n=3,5,7$ and 9 nodes. Solid lines: numerical error. Dashed line: approximate error.

well known that RBF-FD formulas approach standard finite difference formulas [6]. This fact can be clearly observed in the figure which shows how the error approaches the standard finite difference error when $\epsilon \rightarrow 0$.

Notice also that there is a range of values of the shape parameter, $\epsilon$, for which RBF-FD formulas are more accurate than standard finite differences. In particular, there is an optimal value, $\epsilon^{+}$, for which the local truncation error is zero. Since the value of $\epsilon^{+}$can be precisely estimated from the formulas in Table 1, it is possible to use the RBF-FD method to accurately solve PDE problems following the same approach described in references [2,3] for multiquadrics.

The right part of Figure 1 shows the absolute value of the error as a function of the internode distance $h$ for $\epsilon=5$. Notice that the error behaves as $O\left(h^{n-1}\right)$ in agreement with the formulas in Table 1.

\subsection{Second derivative}

Table 2 shows the weights and the corresponding local truncation errors for RBF-FD formulas to approximate the second derivative in $1 \mathrm{D}$ using the standard formulation which is not exact for constants (3). As in the previous case, exact expressions are given for 3,5 and 7 equispaced nodes. 

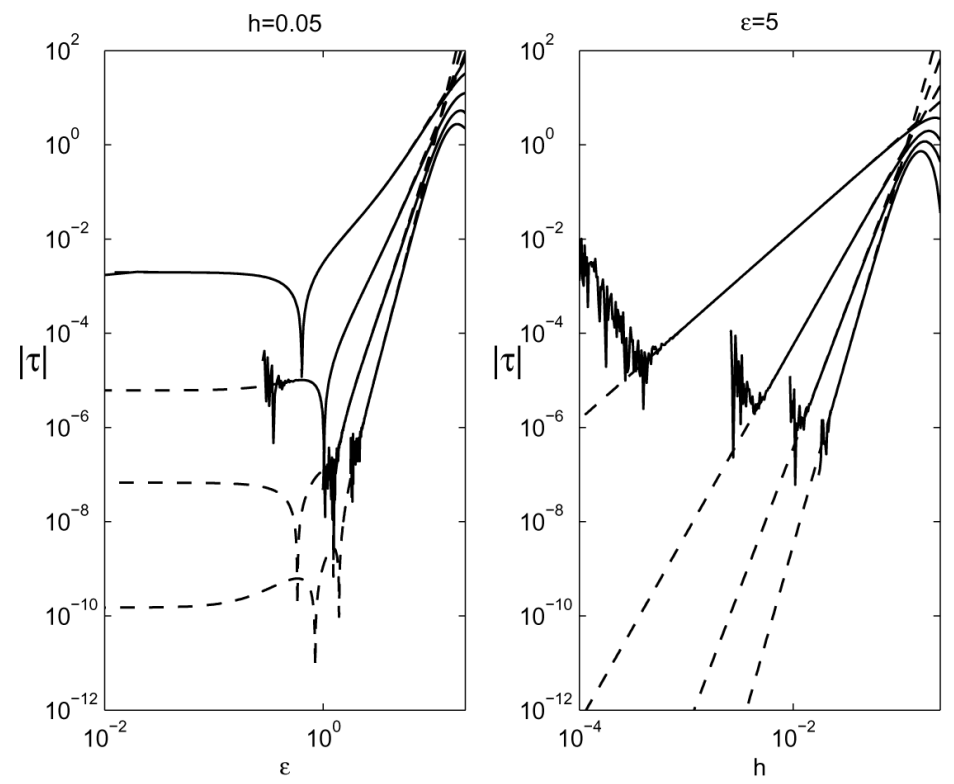

Figure 2: Same as Figure 1 but for the RBF-FD second derivative. Weights computed from equation 3 (non exact for constants).
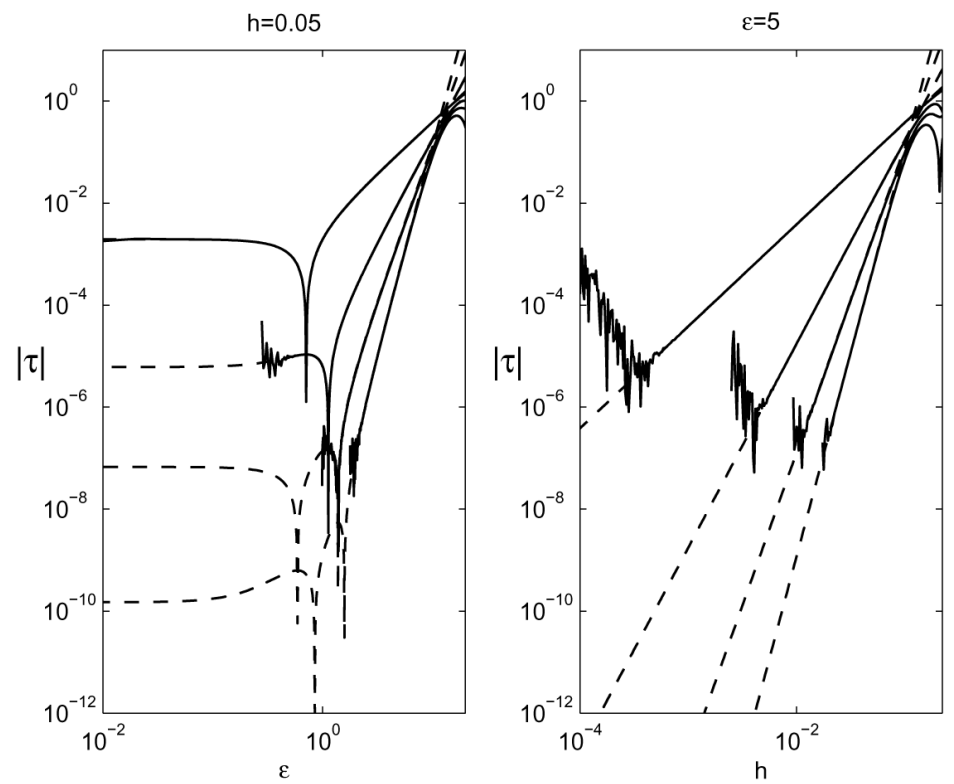

Figure 3: Same as Figure 1 but for the RBF-FD second derivative. Weights computed from equation 4 (exact for constants). 
Table 2: RBF-FD second derivative: non exact for constants.

\begin{tabular}{|c|c|}
\hline \multicolumn{2}{|r|}{ Three nodes } \\
\hline$\alpha_{0}$ & $-2\left(\epsilon^{2}+\epsilon^{4} h^{2} \operatorname{csch}^{2}\left(\epsilon^{2} h^{2}\right)\right)$ \\
\hline$\alpha_{ \pm 1}$ & $\epsilon^{4} h^{2}\left(1+\operatorname{coth}\left(\epsilon^{2} h^{2}\right)\right) \operatorname{csch}\left(\epsilon^{2} h^{2}\right)$ \\
\hline$\tau_{3}$ & $\frac{h^{2}}{12}\left(u^{(I V)}\left(x_{0}\right)+12 \epsilon^{2} u^{\prime \prime}\left(x_{0}\right)+12 \epsilon^{4} u\left(x_{0}\right)\right)+O\left(h^{4} P_{3}\left(\epsilon^{2}\right)\right)$ \\
\hline \multicolumn{2}{|r|}{ Five nodes } \\
\hline$\alpha_{0}$ & $\frac{1}{2}\left(\epsilon^{4} h^{2}\left(\operatorname{sech}^{2}\left(\epsilon^{2} h^{2}\right)-5 \operatorname{csch}^{2}\left(\epsilon^{2} h^{2}\right)\right)-4 \epsilon^{2}\right)$ \\
\hline$\alpha_{ \pm 1}$ & $\frac{4 \epsilon^{4} h^{2} \cosh \left(\epsilon^{2} h^{2}\right) \operatorname{coth}\left(\epsilon^{2} h^{2}\right)\left(\operatorname{coth}\left(\epsilon^{2} h^{2}\right)+1\right)}{2 \cosh \left(2 \epsilon^{2} h^{2}\right)+1}$ \\
\hline$\alpha_{ \pm 2}$ & $-\frac{\epsilon^{4} h^{2} e^{4 \epsilon^{2} h^{2} \operatorname{csch}^{2}\left(2 \epsilon^{2} h^{2}\right)}}{2 \cosh \left(2 \epsilon^{2} h^{2}\right)+1}$ \\
\hline$\tau_{5}$ & $-\frac{h^{4}}{90}\left(u^{(V I)}\left(x_{0}\right)+30 \epsilon^{2} u^{(I V)}\left(x_{0}\right)+180 \epsilon^{4} u^{\prime \prime}\left(x_{0}\right)+120 \epsilon^{6} u\left(x_{0}\right)\right)+O\left(h^{6} P_{4}\left(\epsilon^{2}\right)\right)$ \\
\hline \multicolumn{2}{|r|}{ Seven nodes } \\
\hline$\alpha_{0}$ & $\frac{1}{18} \epsilon^{2}\left(\frac{32 \epsilon^{2} h^{2}\left(\cosh \left(2 \epsilon^{2} h^{2}\right)+2\right)}{\left(2 \cosh \left(2 \epsilon^{2} h^{2}\right)+1\right)^{2}}-49 \epsilon^{2} h^{2} \operatorname{csch}^{2}\left(\epsilon^{2} h^{2}\right)+9 \epsilon^{2} h^{2} \operatorname{sech}^{2}\left(\epsilon^{2} h^{2}\right)-36\right)$ \\
\hline$\alpha_{ \pm 1}$ & $\frac{1}{2} \epsilon^{4} h^{2}\left(\operatorname{coth}\left(\epsilon^{2} h^{2}\right)+1\right) \operatorname{csch}\left(\epsilon^{2} h^{2}\right)\left(\operatorname{sech}\left(2 \epsilon^{2} h^{2}\right)+2\right)$ \\
\hline & $--\frac{\epsilon^{4} h^{2} e^{2 \epsilon^{2} h^{2}}\left(e^{2 \epsilon^{2} h^{2}}+e^{4 \epsilon^{2} h^{2}}+1\right) \operatorname{csch}^{2}\left(2 \epsilon^{2} h^{2}\right)}{}$ \\
\hline$\alpha_{ \pm 2}$ & $-\frac{2 \cosh \left(2 \epsilon^{2} h^{2}\right)+2 \cosh \left(4 \epsilon^{2} h^{2}\right)+1}{2}$ \\
\hline$\alpha_{ \pm 3}$ & $\frac{\epsilon^{4} h^{2} e^{9 \epsilon^{2} h^{2}} \operatorname{csch}^{2}\left(3 \epsilon^{2} h^{2}\right)}{2\left(2 \cosh \left(2 \epsilon^{2} h^{2}\right)+\cosh \left(4 \epsilon^{2} h^{2}\right)+\cosh \left(6 \epsilon^{2} h^{2}\right)+1\right)}$ \\
\hline$\tau_{7}$ & $\frac{h^{6}}{560}\left(u^{(V I I I)}\left(x_{0}\right)+56 \epsilon^{2} u^{(V I)}\left(x_{0}\right)+840 \epsilon^{4} u^{(I V)}\left(x_{0}\right)\right.$ \\
\hline & $\left.+3360 \epsilon^{6} u^{\prime \prime}\left(x_{0}\right)+1680 \epsilon^{8} u\left(x_{0}\right)\right)+O\left(h^{8} P_{5}\left(\epsilon^{2}\right)\right)$ \\
\hline
\end{tabular}

Figure 2 shows the numerical error (solid line) in the approximation of the second derivative with $n=3,5,7$ and 9 using the standard formulation which is not exact for constants (3). The numerical results are compared with the approximate error given by the formulas in Table 2 (dashed line). The left part of Figure 2 shows the absolute value of the error as a function of the shape parameter for $h=0.05$, and the right part shows the absolute value of the error as a function of the internode distance $h$ for $\epsilon=5$. In the first case, the accuracy increases with decreasing $\epsilon$ and approaches standard finite differences for small $\epsilon$. Notice that there is an optimal value, $\epsilon^{+}$, for which the local truncation error is zero. In 
104 Boundary Elements and Other Mesh Reduction Methods XXXIV

Table 3: RBF-FD second derivative: exact for constants.

\begin{tabular}{|c|c|}
\hline \multicolumn{2}{|r|}{ Three nodes } \\
\hline & $4 \epsilon^{2} e^{3 \epsilon^{2} h^{2}}\left(2 \epsilon^{2} h^{2}+e^{\epsilon^{2} h^{2}}-1\right)$ \\
\hline$\alpha_{0}$ & $--4 e^{3 \epsilon^{2} h^{2}}+3 e^{4 \epsilon^{2} h^{2}+1}$ \\
\hline & $2 \epsilon^{2} e^{3 \epsilon^{2} h^{2}}\left(2 \epsilon^{2} h^{2}+e^{\epsilon^{2} h^{2}}-1\right)$ \\
\hline$\alpha_{ \pm 1}$ & $-4 e^{3 \epsilon^{2} h^{2}}+3 e^{4 \epsilon^{2} h^{2}+1}$ \\
\hline$\tau_{3}$ & $\frac{h^{2}}{12}\left(u^{(I V)}\left(x_{0}\right)+10 \epsilon^{2} u^{\prime \prime}\left(x_{0}\right)\right)+O\left(h^{4} P_{2}\left(\epsilon^{2}\right)\right)$ \\
\hline \multicolumn{2}{|r|}{ Five nodes } \\
\hline$\alpha_{0}$ & $-\frac{5}{2 h^{2}}-\frac{28 \epsilon^{2}}{15}+\frac{83 h^{2} \epsilon^{4}}{90}+O\left(h^{4} \epsilon^{6}\right)$ \\
\hline$\alpha_{ \pm 1}$ & $\frac{4}{3 h^{2}}+\frac{56 \epsilon^{2}}{45}-\frac{13 h^{2} \epsilon^{4}}{135}+O\left(h^{4} \epsilon^{6}\right)$ \\
\hline$\alpha_{ \pm 2}$ & $-\frac{1}{12 h^{2}}-\frac{14 \epsilon^{2}}{45}-\frac{197 h^{2} \epsilon^{4}}{540}+O\left(h^{4} \epsilon^{6}\right)$ \\
\hline$\tau_{5}$ & $-\frac{h^{4}}{90}\left(u^{(V I)}\left(x_{0}\right)+28 \epsilon^{2} u^{(I V)}\left(x_{0}\right)+140 \epsilon^{4} u^{\prime \prime}\left(x_{0}\right)\right)+O\left(h^{6} P_{3}\left(\epsilon^{2}\right)\right)$ \\
\hline \multicolumn{2}{|r|}{ Seven nodes } \\
\hline$\alpha_{0}$ & $-\frac{49}{18 h^{2}}-\frac{27 \epsilon^{2}}{14}+\frac{237 h^{2} \epsilon^{4}}{140}+\frac{199 h^{4} \epsilon^{6}}{300}+O\left(h^{6} \epsilon^{8}\right)$ \\
\hline$\alpha_{ \pm 1}$ & $\frac{3}{2 h^{2}}+\frac{81 \epsilon^{2}}{56}-\frac{333 h^{2} \epsilon^{4}}{560}-\frac{533 h^{4} \epsilon^{6}}{400}+O\left(h^{6} \epsilon^{8}\right)$ \\
\hline$\alpha_{ \pm 2}$ & $-\frac{3}{20 h^{2}}-\frac{81 \epsilon^{2}}{140}-\frac{801 h^{2} \epsilon^{4}}{1400}+\frac{127 h^{4} \epsilon^{6}}{200}+O\left(h^{6} \epsilon^{8}\right)$ \\
\hline$\alpha_{ \pm 3}$ & $\frac{1}{90 h^{2}}+\frac{27 \epsilon^{2}}{280}+\frac{897 h^{2} \epsilon^{4}}{2800}+\frac{439 h^{4} \epsilon^{6}}{1200}+O\left(h^{6} \epsilon^{8}\right)$ \\
\hline$\tau_{7}$ & $\frac{h^{6}}{560}\left(u^{(V I I I)}\left(x_{0}\right)+54 \epsilon^{2} u^{(V I)}\left(x_{0}\right)\right.$ \\
\hline & $\left.+756 \epsilon^{4} u^{(I V)}\left(x_{0}\right)+2520 \epsilon^{6} u^{\prime \prime}\left(x_{0}\right)\right)+O\left(h^{8} P_{4}\left(\epsilon^{2}\right)\right)$ \\
\hline
\end{tabular}

the second case, the error behaves as $O\left(h^{n-1}\right)$ in agreement with the formulas in Table 2 .

Table 3 shows the weights and the corresponding local truncation errors for RBF-FD formulas to approximate the second derivative in $1 \mathrm{D}$ using the standard formulation which is exact for constants (4). In this case, exact expressions are only given for 3 equispaced nodes. For 5,7 and 9 equispaced nodes we only include their series expansions in the limit $\epsilon h \ll 1$. Figure 3 shows the corresponding error (solid line) for $n=3,5,7,9$, and compares it with the approximate error given 

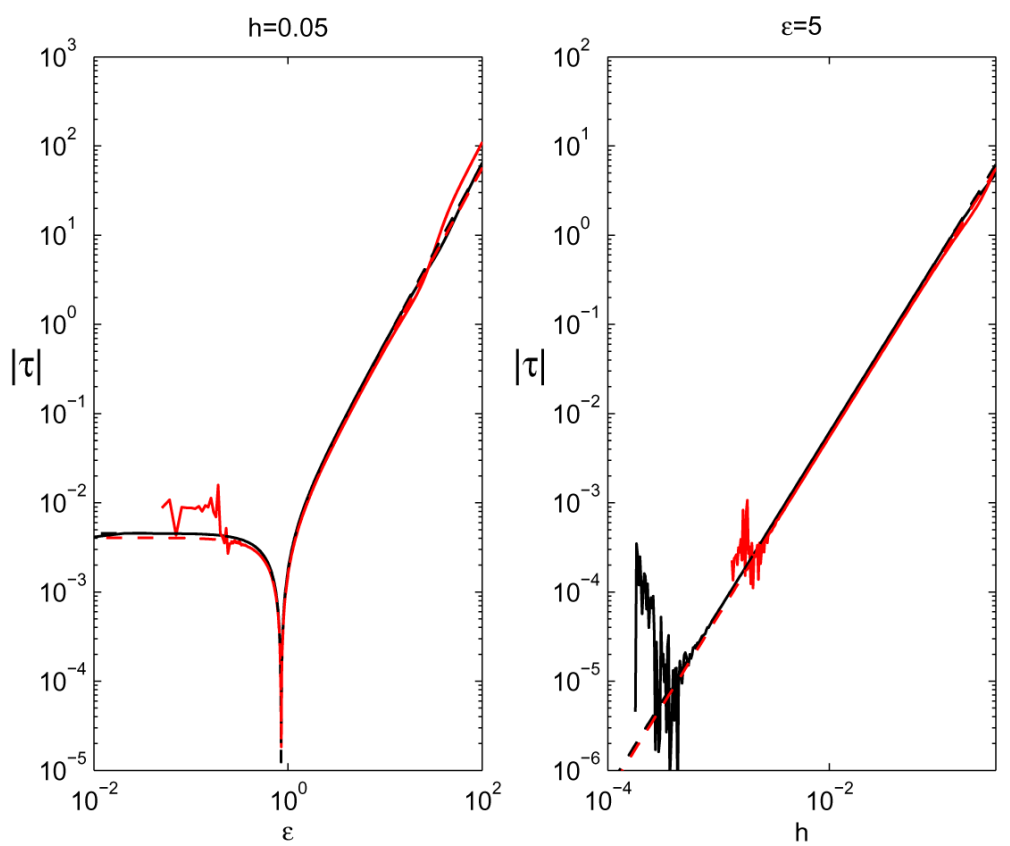

Figure 4: Local truncation error $\tau$ for the RBF-FD Laplacian (exact for constants) as function of $\epsilon$ (left side) and $h$ (right side) using structured stencils with $n=5$ (black) and 9 (red) nodes. Solid lines: numerical error. Dashed line: analytical error.

by the formulas in table 3 (dashed line). Both results coincide until the system of equations (3) becomes ill-conditioned. As in the previous cases, the existence of an optimal shape parameter, $\epsilon^{+}$which makes the error zero, can be clearly observed.

For $h \ll 1$ the error resulting from the formulation which is exact for constants (4) and from the formulation that is not exact (3) coincide (see figures 2 and 3 and tables 2 and 3). Notice however, that the error corresponding to the formulation which is non exact for constants (table 2) contains some extra terms. For instance, in the case of three nodes, the error for the non exact case includes a term proportional to $\epsilon^{4}$ while the error corresponding to the exact case does not (table 3 ). Thus, for values of $\epsilon$ of order unity or larger, the two formulations may differ significantly.

\subsection{Laplacian}

Table 4 shows the weights and the corresponding local truncation errors for the RBF-FD laplacian formulas in the case which is exact for constants. To simplify the formulas we have used $w=\epsilon^{2} h^{2}$. Figure 4 shows the corresponding errors (solid lines) for $n=5$ (black) and 9 (red), and compares them with the approximate errors given in the table (dashed line). 
106 Boundary Elements and Other Mesh Reduction Methods XXXIV

Table 4: RBF-FD Laplacian: exact for constants.

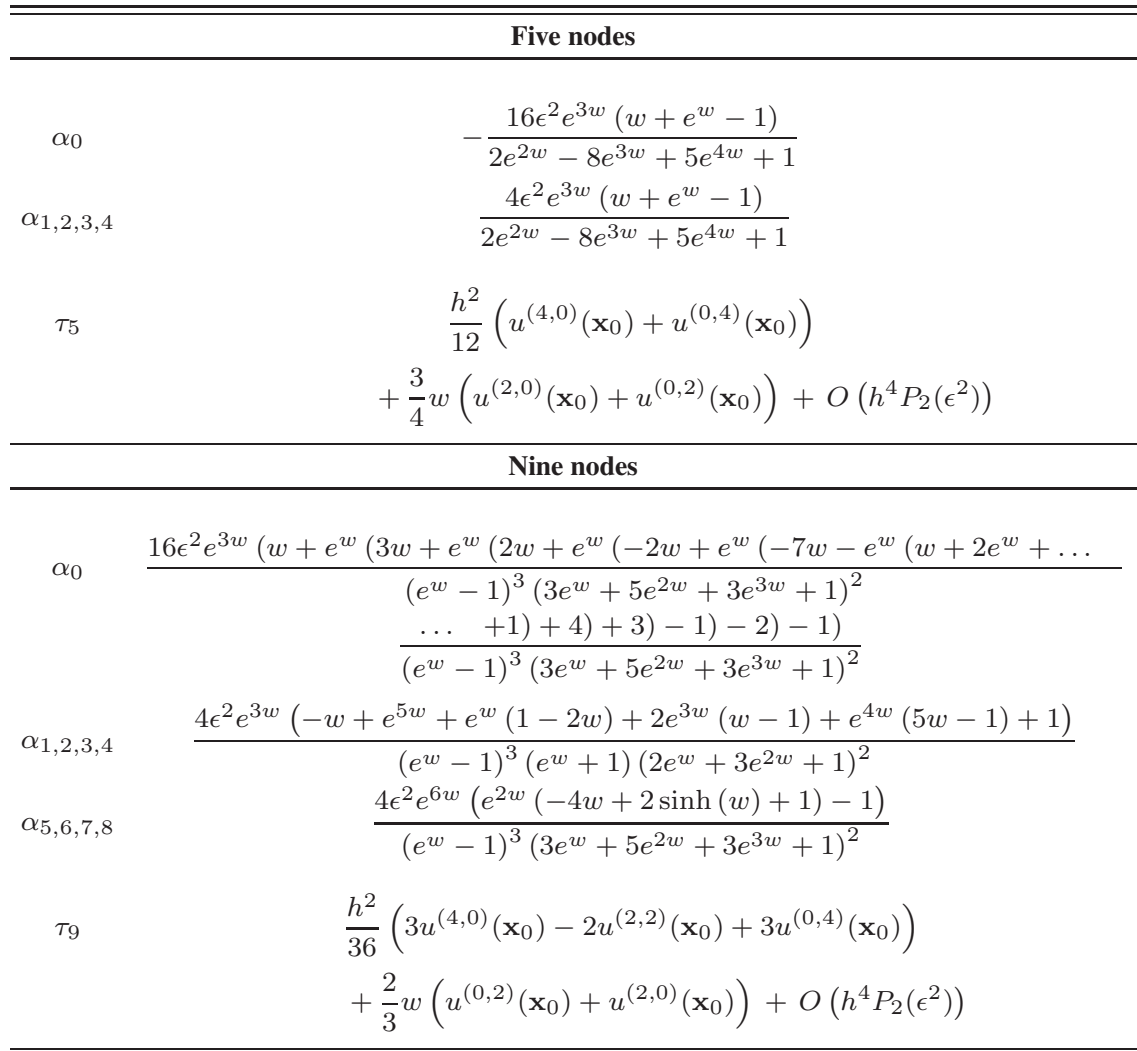

Notice that the weights and the error for the 5 nodes case are different from those of the 9 node case. However, it can be observed in figure 4 that, although the formulas for the error are different for 5 and 9 nodes, the actual numerical error with 5 and 9 nodes are quite similar in the range of parameters chosen. Notice also that ill-conditioning is also more severe with 9 nodes than with 5 nodes.

Notice also that, in both the exact and non-exact cases, there exist an optimal shape parameter which makes the error zero. For the non-exact case $\epsilon^{+} \approx 0.7019$ for both 5 and 9 nodes. For the exact case $\epsilon^{+} \approx 0.8469$ for five nodes and $\epsilon^{+} \approx 0.8481$ for nine nodes.

\section{Conclusions}

In this work we derive analytical expressions for the weights of RBF-FD formulas for first and second derivatives in 1D, and for the Laplacian in 2D using Gaussians 
as RBFs. Results are presented for 3, 5, 7 and 9 nodes in 1D, and for 5 and 9 nodes in the case of RBF-FD formulas in $2 \mathrm{D}$. These weights are then used to derive analytical expressions for the leading order approximations to the local error in powers of the internode distance $h$. We show that the agreement of these formulas with the actual numerical error is very good.

We also show that for each differential operator, there is a range of values of the shape parameter for which RBF-FD formulas are significantly more accurate than the corresponding conventional finite difference formulas. In fact, very often there is an optimal value of the shape parameter $\epsilon^{+}$for which the error is zero. This value can be easily computed from the analytical expressions for the leading order approximations to the local error. Contrary to what is generally believed, this value is, to leading order, independent of the internodal distance and only dependent on the value of the function and its derivatives at the node.

The results presented in this paper can be used to efficiently solve PDE problems using RBF-FD formulas, by selecting a constant optimal value of the shape parameter (as was done in [2] for multiquadrics) or by selecting a node-dependent optimal shape parameter (as was done in [3] for multiquadrics).

It should be also emphasized that, contrary to what happened with multiquadrics [1], for Gaussians it is often possible to write the weights as exact analytical formulas. Thus, it is not necessary to numerically solve the linear system defining the weights and, thereby, the problem of ill-conditioning which appears often when using these techniques, can be completely avoided.

\section{References}

[1] V. Bayona, M. Moscoso, M. Carretero, M. Kindelan, RBF-FD formulas and convergence properties, J. Comput. Phys. 229, (2010) 8281-8295.

[2] V. Bayona, M. Moscoso, M. Kindelan, Optimal constant shape parameter for multiquadric based RBF-FD method, J. Comput. Phys. accepted (2011).

[3] V. Bayona, M. Moscoso, M. Kindelan, Optimal (variable) shape parameter for multiquadric based RBF-FD method, J. Comput. Phys. submitted (2011).

[4] J. P. Boyd, L. Wang, Truncated Gaussian RBF Differences are Always Inferior to Finite Differences of the Same Stencil Width, Commun. Comput. Phys., 5 (2009),42-60.

[5] L. Collatz, The numerical treatment of differential equations, Springer, Berlin (1960).

[6] T. A. Driscoll, B. Fornberg, Interpolation in the limit of increasingly flat radial basis functions, Comput. Math. Appl. 43 (2002) 413-422.

[7] G. E. Fasshauer, Meshfree Approximation Methods with MATLAB, World Scientific Publishing Co., Singapore (2007).

[8] O. Davydov and D. T. Oanh, Adaptive meshless centers and RBF stencils for Poisson equation, J. Comput. Phys. 230 (2011) 287-304.

[9] O. Davydov and D. T. Oanh, On Optimal Shape Parameter for Gaussian RBFFD Approximation of Poisson Equation, Univ. of Strathclyde Report (2011). 
[10] N. Flyer, G. B. Wright, Transport Schemes on a Sphere Using Radial Basis Functions, J. Comput. Phys. 226 (2007) 1059-1084.

[11] B. Fornberg, E. Letho, Stabilization of RBF-generated finite difference methods for convective PDEs, J. Comput. Phys. 230 (2011) 2270-2285.

[12] E. J. Kansa, Multiquadrics, a scattered data approximation scheme with applications to computational fluid dynamics. I. Surface approximations and partial derivatives estimates, Comput. Math. Appl. 19 (1990) 127-145.

[13] E. J. Kansa, Multiquadrics, a scattered data approximation scheme with applications to computational fluid dynamics. II. Solutions to parabolic, hyperbolic and elliptic partial differential equations, Comput. Math. Appl. 19 (1990) 147-161.

[14] B. Seibold, Minimal positive stencils in meshfree finite difference methods, Comput. Methods Appl. Mech. Engrg. 198 (2008) 592-601.

[15] C. Shu, H. Ding, K.S. Yeo, Local radial basis function-based differential quadrature method and its application to solve two-dimensional incompressible Navier-Stokes equations, Computer Methods in Applied Mechanics and Engineering 192 (2003) 941-954.

[16] A. I. Tolstykh and D. A. Shirobokov, On using radial basis functions in a "finite difference mode" with applications to elasticity problems, Computational Mechanics 33, (2003) 68-79.

[17] G. B. Wright, Radial basis function interpolation: numerical and analytical developments, Ph.D. thesis, University of Colorado, Boulder (2003).

[18] G. B. Wright, B. Fornberg, Scattered node compact finite difference-type formulas generated from radial basis functions, J. Comput. Phys. 212, (2006) 99-123. 